\title{
Emerging roles of let-7d in attenuating pulmonary arterial hypertension via suppression of pulmonary artery endothelial cell autophagy and endothelin synthesis through ATG16L1 downregulation
}

\author{
MINGHUI OU ${ }^{1}$, XIA LI $^{2}$, SHICHAO CUI $^{1}$, SHIBO ZHAO $^{1}$ and JIE TU $^{3}$ \\ Departments of ${ }^{1}$ Vascular Surgery, ${ }^{2}$ Ultrasound, and ${ }^{3}$ Science and Education, Qingdao Municipal Hospital, \\ Qingdao, Shandong 266011, P.R. China
}

Received January 11, 2019; Accepted November 8, 2019

DOI: $10.3892 /$ ijmm.2020.4567

\begin{abstract}
Pulmonary arterial hypertension (PAH) is a severe disease characterized by elevated pulmonary arterial pressure and pulmonary vascular resistance, resulting in right ventricular failure and death. Compelling evidence has suggested the roles of microRNAs (miRNAs/miRs) in PAH. The present study investigated the possible effects of miR-let-7d on PAH through autophagy-related 16-like 1 (ATG16L1). Initially, the serum levels of let-7d in PAH patients were detected. Rats were then treated with monocrotaline to induce a rat model of PAH, after which the right ventricular systolic pressure (RVSP) and right ventricular hypertrophy index (RVHI) were determined. Next, the putative binding sites between let-7d and ATG16L1 were detected. The expression of let-7d and ATG16L1 in PAH rat models and cells was upregulated or downregulated to assess the effects of these molecules on autophagy in pulmonary artery vascular endothelial cells (PAECs) and on endothelin synthesis. In addition, the levels of p62, LC3-I, LC3-II, LC3B and endothelin-1 (ET-1) were assessed. The results obtained revealed that let-7d was downregulated in the serum of PAH patients and rats with PAH. Importantly, ATG16L1 was found to be a target gene of let-7d and let-7d could suppress the expression of ATG16L1. Overexpression of let-7d was found to reduce RVSP and RVHI values. Additionally, upregulation of let-7d or depletion of ATG16L1 led to suppression of PAEC autophagy and endothelin synthesis, corresponding to decreased ratios of LC3-II to LC3-I and reduced levels of LC3B but elevated levels of p62 in PAECs and ET-1 in plasma and lung tissues. In summary, let-7d upregulation alleviates
\end{abstract}

Correspondence to: Dr Jie Tu, Department of Science and Education, Qingdao Municipal Hospital, 1 Jiaozhou Road, Qingdao, Shandong 266011, P.R. China

E-mail: tujie3200@163.com

Key words: pulmonary arterial hypertension, let-7d, endothelin synthesis, autophagy-related 16-like 1, pulmonary artery endothelial cells, autophagy
PAH by inhibiting autophagy in PAECs and suppressing endothelin synthesis through negative regulation of ATG16L1.

\section{Introduction}

The progressive disease pulmonary arterial hypertension (PAH) is characterized by increased pulmonary arterial pressure and pulmonary vascular resistance, leading to right ventricular failure and eventually death (1). The pathophysiology of PAH is characterized by vascular remodeling and a vasoconstrictive and proliferative thrombotic phenotype (2). Patients suffering from PAH always experience symptoms such as exertional dyspnea, fatigue, chest pain and dizziness (3). It has been found that proliferation of pulmonary artery endothelial cells (PAECs) and pulmonary artery smooth muscle cells (PASMCs) contributes to the obstruction of the vascular lumen in the late stage (4). Although progress has been made in PAH treatment in recent years, PAH remains a disease with limited treatment modalities (5). Most patients still have low overall survival rates and their quality of life remains severely affected (6). Therefore, a deeper understanding of the molecular mechanisms of PAH is needed to identify effective therapeutic targets for this complex disease (7).

MicroRNAs (miRNAs/miR) are engaged in the regulation of various biological processes, such as cell differentiation, proliferation and apoptosis, and dysregulation of miRNAs is involved in various human diseases (8). Recently, evidence has emerged showing that abnormal expression of miRNAs participates in the biological development of PAH $(9,10)$. Let-7 family members have been demonstrated to be key regulators of cell development and differentiation and their tumor-suppressive roles have been found in various human cancers (11). For example, miR-let-7d is a member of the let-7 family and its upregulation can inhibit cell proliferation and migration and promote apoptosis of trophoblast cells in preeclampsia (12). Most importantly, the let-7 family has also been demonstrated to be abnormally expressed in cardiovascular diseases, such as heart hypertrophy, dilated cardiomyopathy and hypertension (13). Autophagy plays an important role in cardiovascular cells (14) and miRNAs have been shown to function vitally in the regulation of autophagy-related pathways (15). In addition, 
autophagy-related 16-like 1 (ATG16L1) has been identified as an autophagy-related gene that can regulate autophagosomes (16). ATG16L1 belongs to a class of protein complexes that are considered vital for autophagy (17). Emerging data has illustrated the functionality of ATG16L1 in the development of atherogenesis (18). Based on the aforementioned findings, it has been demonstrated that both let-7d and ATG16L1 may participate in PAH by affecting autophagy. The present study further hypothesized that let-7d may interact with ATG16L1 to participate in $\mathrm{PAH}$ and the present study was performed to elucidate this interaction.

\section{Materials and methods}

Ethics statement. The current study was approved by the Ethics Committee and the Experimental Animal Ethics Committee of Qingdao Municipal Hospital. Written informed consent was obtained from all participants prior to the study. The animal experiment strictly adhered to the principles of using the least number of animals to complete the experiment and minimizing the pain of the experimental animals.

Patient enrollment. A total of 83 PAH patients hospitalized at the Qingdao Municipal Hospital between June 2016 and June 2018 were selected for the study. The patients were included if their pulmonary artery systolic pressure was $>30 \mathrm{mmHg}$ as detected by echocardiography. The patients were excluded if they suffered from cardiomyopathy, myocardial infarction, heart failure, valvular disease, pericardial disease, chronic thromboembolic disease, or chronic obstructive pulmonary disease. All included patients (51 males and 32 females), aged 19-70 years with an average age of 45.70 \pm 13.89 years, had complete clinical data and did not receive any surgeries or drug treatment prior to the operation $(19,20)$. A total of 40 healthy individuals were enrolled as the normal group. Patient characteristics are presented in Table I. The levels of let-7d in $2 \mathrm{ml}$ plasma collected (stored at $-80^{\circ} \mathrm{C}$ ) from each PAH patients and healthy individuals were measured.

Rat models simulating $P A H$. In total, 85 male healthy specific-pathogen-free grade Sprague-Dawley rats (age, 7 weeks; weighing 220-250 g) were purchased from the Qingdao Municipal Hospital Experimental Animal Center. All animals were maintained at $22 \pm 2^{\circ} \mathrm{C}$ with a humidity of $55 \pm 5 \%$, a 12:12 h light/dark cycle and free access to regular mouse chow and water for 1 week. Monocrotaline (MCT; Sigma-Aldrich; Merck KGaA) was dissolved in $0.1 \mathrm{~mol} / 1 \mathrm{HCl}$ and titrated with $0.1 \mathrm{~mol} / 1 \mathrm{NaOH}$ to a $\mathrm{pH}$ of 7.4 and a final concentration of $30 \mathrm{mg} / \mathrm{ml}$; the final solution $(60 \mathrm{mg} / \mathrm{kg})$ was then injected intraperitoneally into the rats of the PAH group. The rats in the control group were injected with equal volumes of normal saline. After 28 days of MCT induction, the rats in the PAH group presented with significant PAH symptoms compared with those in the control group. A lentiviral vector was constructed as previously reported (21); the ATG16L1 gene sequence was amplified by PCR and then the amplified product was cloned into the lentiviral gene overexpression vector pLV-EGFP-N at the EcoRI and NotI sites using a Cold Fusion kit (System Biosciences LLC). Cells were infected with empty lentiviral particles or lentiviral overexpression particles and selected with puromycin for 3 days to obtain stable cell lines. Both the overexpressed (oe)-negative control (NC) and oe-ATG16L1 vectors were constructed by Shanghai GenePharma Co., Ltd. 293 T cells (American Type Culture Collection) were used for lentiviral packaging and the $293 \mathrm{~T}$ cells were cultured in RPMI-1640 complete medium (Gibco; Thermo Fisher Scientific, Inc.) containing 10\% fetal bovine serum (FBS; Gibco; Thermo Fisher Scientific, Inc.), followed by sub-culture every other day. The viruses $\left(1.0 \times 10^{8} \mathrm{PFU} / \mathrm{ml}\right)$ were collected and the cells were infected with oe-ATG16L1 plasmid or NC plasmid. Rats were anesthetized with $3 \%$ sodium pentobarbital (P3761; Sigma-Aldrich; Merck KGaA) 28 days after MCT induction. The rats were then fixed on a sterilized test bench and the tail of each rat was repeatedly rubbed with a cotton ball soaked in alcohol. After the veins on both sides of the tail were dilated, the residual air bubbles in the syringe were removed and adenoviral vector $\left(1 \times 10^{9} \mathrm{PFU} / 100 \mu \mathrm{l}\right)$, let- $7 \mathrm{~d}$ agomir $(20 \mathrm{nM})$, or let-7d antagomir $(20 \mathrm{nM})$ was injected into the dilated tail vein at the proximal end at a $30^{\circ}$ angle. After infection for $48 \mathrm{~h}$, green fluorescent protein expression efficiency was observed under a fluorescence microscope and relevant experiments were performed.

The modeling success was assessed using right ventricular systolic pressure (RVSP) by right heart catheterization, right ventricular hypertrophy with right ventricular hypertrophy index (RVHI), and the morphology of the pulmonary vessels by hematoxylin and eosin (H\&E) staining. A total of 10 normal rats were taken as the normal group. Among the remaining 75 rats, the model was successfully induced in 70, with a modeling success rate of $93.33 \%$. The PAH rat models were assigned into 7 groups with 10 rats in each group: The PAH group (PAH rat models), the agomir-NC PAH group (PAH rat models injected with agomir-NC), the let-7d agomir PAH group (PAH rat models injected with let-7d agomir), the antagomir-NC PAH group (PAH rat models injected with antagomir-NC), the let-7d antagomir PAH group (PAH rat models injected with let-7d antagomir), the let-7d agomir + oe-NC PAH group (PAH rat models injected with let-7d agomir and oe-NC lentivirus) and the let-7d agomir + oe-ATG16L1 PAH group (PAH rat models injected with let-7d agomir and oe-ATG16L1 lentivirus). The rats were euthanized by deep anesthesia with pentobarbital sodium $(100 \mathrm{mg} / \mathrm{kg})$ on the 28 th day after MCT induction $48 \mathrm{~h}$ after injection of agomir/antagomir or lentivirus.

Determination of RVSP and RVHI. RVSP and the RVHI were measured on the 28th day of MCT induction $48 \mathrm{~h}$ after injection of the agomir/antagomir or lentiviruses. Each rat was anesthetized with $3 \%$ pentobarbital sodium. The right external jugular vein was cannulated and the superior vena cava, right atrium, right ventricle, and pulmonary artery were connected to a physiological polygraph (PowerLab; ADInstruments). The location of the catheter was judged from the pressure waveform changes displayed on the polygraph. After the abdomen was opened, the left ventricle and right ventricle were visualized through the complete diaphragm. Next, a $23 \mathrm{G}$ needle was inserted into the right ventricle, and RVSP was recorded. Subsequently, hemodynamic data were collected and the heart was isolated with the atria and major blood vessels removed. Dissection of the right ventricle (RV) from the left ventricle (LV) and the septum (S) was performed. The weights of the 
Table I. Relationship between let-7d expression and clinicopathological features of PAH patients.

Let-7d expression

\begin{tabular}{|c|c|c|c|c|}
\hline \multirow[b]{2}{*}{ Clinicopathological features } & \multirow[b]{2}{*}{ Case $(n=83)$} & & \multirow[b]{2}{*}{ P-value } \\
\hline & & $\begin{array}{c}\text { Low } \\
\text { expression }(n=39)\end{array}$ & $\begin{array}{c}\text { High } \\
\text { expression }(n=44)\end{array}$ & \\
\hline Age & & & & 0.805 \\
\hline$<50$ years & 35 & 17 & 18 & \\
\hline$\geq 50$ years & 48 & 22 & 26 & \\
\hline Sex & & & & 0.987 \\
\hline Male & 51 & 24 & 27 & \\
\hline Female & 32 & 15 & 17 & \\
\hline Cardiac function grade & & & & 0.003 \\
\hline I & 28 & 9 & 19 & \\
\hline II & 32 & 12 & 20 & \\
\hline III & 13 & 9 & 4 & \\
\hline IV & 10 & 9 & 1 & \\
\hline Pulmonary hypertension degree & & & & 0.021 \\
\hline Mild & 34 & 10 & 24 & \\
\hline Middle & 26 & 14 & 12 & \\
\hline Severe & 23 & 15 & 8 & \\
\hline Six-min walk test performance classification & & & & 0.019 \\
\hline Class $1(<150 \mathrm{~m})$ & 15 & 3 & 12 & \\
\hline Class $2(150-425 \mathrm{~m})$ & 49 & 23 & 26 & \\
\hline Class $3(426-550 \mathrm{~m})$ & 19 & 13 & 6 & \\
\hline
\end{tabular}

PAH, pulmonary arterial hypertension.

$\mathrm{RV}$ and the $\mathrm{LV}+\mathrm{S}$ were determined on an electronic balance and the RL gravity was expressed as $\mathrm{LV}+\mathrm{S}$.

$H \& E$ staining. The lung tissues were fixed with $4 \%$ paraformaldehyde at room temperature for $12-24 \mathrm{~h}$, paraffin-embedded and cut into $5 \mu \mathrm{m}$ sections. The sections were deparaffinized using xylene I for $10 \mathrm{~min}$ and xylene II for $5 \mathrm{~min}$. Then, the tissues were washed with anhydrous alcohol to remove the xylene for $1 \mathrm{~min}$, with $95 \%$ alcohol for $1 \mathrm{~min}$ and with $85 \%$ alcohol for $1 \mathrm{~min}$. After being washed with tap water, the sections were stained with hematoxylin (Beyotime Institute of Biotechnology) for $5 \mathrm{~min}$ at room temperature, followed by an additional wash with tap water. Upon removal of the water, the sections were differentiated with hydrochloric acid ethanol, soaked in water for $15 \mathrm{~min}$ and stained with eosin solution (Beyotime Institute of Biotechnology) for $2 \mathrm{~min}$ at room temperature before being dehydrated with $85 \%$ alcohol for $20 \mathrm{sec}$ and $95 \%$ alcohol for $1 \mathrm{~min}$. After that, the sections were incubated with anhydrous alcohol I and anhydrous alcohol II for 2 min each and soaked in xylene I and xylene II for 2 min each. The sections were then mounted with neutral balsam or Canadian balsam and observed under a light microscope (DMI3000, Leica $\mathrm{GmbH}$ ) after H\&E staining. To assess vascular remodeling, the percentage of medial wall thickness (\% MT; diameter 50-250 $\mu \mathrm{m}$ ) was calculated using the following formula: $\mathrm{MT}=(2 \mathrm{x}$ medial wall thickness $)$ x100/outer diameter.
Human PAEC culture and treatment. PAECs were purchased from Lonza Group, Ltd. In strict accordance with the protocol, the cells were cultured with $10 \%$ FBS and high-glucose Dulbecco's modified eagle medium (DMEM; Invitrogen; Thermo Fisher Scientific, Inc.) and observed under a light microscope. The cells at passage 3 were detached with trypsin, inoculated into a 24 -well plate at a density of $2 \times 10^{6}$ cells/well and cultured until they grew into monolayers. When the cell density reached $75 \%$, the cells were considered to be in logarithmic phase and were inoculated into a 6-well cell culture plate. After $12 \mathrm{~h}$, according to the protocol of Lipofectamine 2000 (Invitrogen; Thermo Fisher Scientific, Inc.), the cells were transfected with $\mathrm{NC}$ mimic, let-7d mimic, NC inhibitor or let-7d inhibitor plasmids (final concentration, $50 \mathrm{nM}$ ) for $48 \mathrm{~h}$ (22). The cells were then assigned to the following groups: The blank group (without transfection of any plasmid), the NC mimic group (transfected with NC mimic plasmid), the let-7d mimic group (transfected with let- $7 \mathrm{~d}$ mimic plasmid), the $\mathrm{NC}$-inhibitor group (transfected with NC inhibitor plasmid) and the let-7d inhibitor group (transfected with let-7d inhibitor plasmid). The transfection plasmid was constructed by Invitrogen; Thermo Fisher Scientific, Inc.

Dual luciferase reporter gene assay. The biological prediction website microRNA.org was employed to analyze the target genes of let-7d and to verify whether ATG16L1 was a direct target gene of let-7d. A dual luciferase reporter gene assay was 
Table II. Primer sequences for reverse transcription-quantitative PCR.

\begin{tabular}{ll}
\hline Gene & \multicolumn{1}{c}{ Primer sequence } \\
\hline Let-7d (Rattus norvegicus) & F: 5'-GCGAGCACAGAATTAATACGAC-3' \\
ATG16L1 (Homo sapiens) & R: 5'-AGAGGTAGTAGGTTGCATAGTT-3' \\
& F: 5'-CAGTTACGTG GCGGCAGGCT-3' \\
U6 (Rattus norvegicus) & R: 5'-ACAACGTGCG AGCCAGAGGG-3' \\
& F: 5'-CTCGCTTCGGCAGCA-3' \\
$\beta$-actin (Homo sapiens) & R: 5'-AACGCTTCACGAATTTGCGT-3' \\
& F: 5'-TGGCACCCAGCACAATGAA-3' \\
& R: 5'-CTAAGTCATAGTCCGCCTAGAAGCA-3'
\end{tabular}

ATG16L1, autophagy-related 16-like 1; F, forward; R, reverse.

employed to confirm that ATG16L1 was a direct target gene of let-7d. A synthesized ATG16L1 3' untranslated region (3'UTR) gene fragment was introduced into the pMIR reporter (Beijing Huayueyang Biotechnology Co., Ltd.) using the endonuclease sites SpeI and HindIII. A complementary sequence with a mutation at the site of the seed sequence was designed on the ATG16L1-wild type (Wt) sequence and the target fragment was inserted into the pMIR reporter plasmid by restriction endonuclease digestion using T4 DNA ligase. The Wt and mutant (Mut) luciferase reporter plasmids with the correct sequences were cotransfected into $293 \mathrm{~T}$ cells with let-7d mimic or let-7d NC (Shanghai Beinuo Biotechnology Co., Ltd.) with Attractene Transfection reagent (cat. no. 301005; Qiagen $\mathrm{GmbH}$ ). After $48 \mathrm{~h}$ of transfection, the cells were collected and lysed, and luciferase activity was measured using a GloMax 20/20 luminometer (Promega Corporation) and a luciferase assay kit (K801-200; BioVision). Firefly luciferase activity was normalized to Renilla luciferase activity.

RNA isolation and quantitation. Reverse transcription-quantitative PCR (RT-qPCR) was employed to detect let-7d expression and ATG16L1 mRNA expression in tissues or cells. Total RNA was extracted using TRIzol (Invitrogen; Thermo Fisher Scientific, Inc.) from the lung tissues of rats $48 \mathrm{~h}$ after the different injections were administered and PAECs were collected $24 \mathrm{~h}$ after transfection. The sequences of the primers are shown in Table II. The primers of let-7d and ATG16L1 were designed and synthesized by Invitrogen (Thermo Fisher Scientific, Inc.). Total RNA was reverse transcribed into complementary DNA (cDNA) using different reverse transcription kits, including a TaqMan ${ }^{\mathrm{TM}}$ MicroRNA Reverse Transcription kit (4366596; Thermo Fisher Scientific Inc.) and a High-Capacity cDNA Reverse Transcription kit (4368813; Thermo Fisher Scientific, Inc.) The temperature protocol for RT was as follows: $42^{\circ} \mathrm{C}$ for $15 \mathrm{~min}, 85^{\circ} \mathrm{C}$ for $5 \mathrm{sec}$ and storage at $4^{\circ} \mathrm{C}$. With U6 and $\beta$-actin used as internal controls (Invitrogen; Thermo Fisher Scientific, Inc.), the PCR system was set to a $25 \mu \mathrm{l}$ volume using a qPCR kit (Takara Bio, Inc.) on a real-time fluorescence quantitative PCR instrument (Thermo Fisher Scientific, Inc.) for PCR. The qPCR program consisted of initial denaturing at $95^{\circ} \mathrm{C}$ for $2 \mathrm{~min}$, followed by 45 cycles of $15 \mathrm{sec}$ at $95^{\circ} \mathrm{C}$ and $45 \mathrm{sec}$ at $60^{\circ} \mathrm{C}$. The final data were analyzed by the $2^{-\Delta \Delta \mathrm{Cq}}$ method (23).

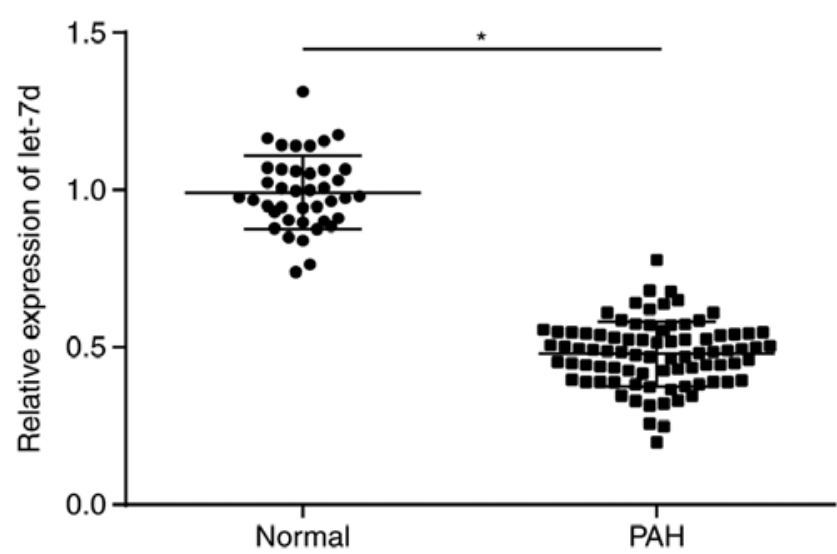

Figure 1. Expression of let-7d is decreased in the plasma of PAH patients, as detected by reverse transcription-quantitative PCR. ${ }^{*} \mathrm{P}<0.05$ vs. the normal group. The data were measurement data and expressed as the mean \pm standard deviation. Differences between two groups were analyzed using independent sample t-test. PAH, pulmonary arterial hypertension.

Western blot analysis. Rat lung tissues were washed with PBS and incubated with RIPA protein lysis buffer(Beyotime Institute of Biotechnology) containing protease and alkaline phosphatase inhibitors for $30 \mathrm{~min}$ at $4^{\circ} \mathrm{C}$. The lysate was collected in a $1.5 \mathrm{ml}$ eppendorf tube and centrifuged at $6,700 \mathrm{x} \mathrm{g}$ for $15 \mathrm{~min}$, after which the supernatant was collected. Then, the supernatant was mixed with loading dye and boiled for $5 \mathrm{~min}$. Protein concentration was measured using a bicinchoninic acid assay kit (Beyotime Institute of Biotechnology). Next, the protein $(50 \mu \mathrm{g})$ was separated using 10\% SDS-PAGE and transferred onto a polyvinylidene fluoride membrane at $0.3 \mathrm{~A}$ and $20 \mathrm{~V}$, which was then blocked with $5 \%$ skim milk powder for $1 \mathrm{~h}$ at room temperature. After that, the membrane was incubated overnight at $4^{\circ} \mathrm{C}$ with the following primary antibodies diluted in Tris-buffered saline with $0.1 \%$ Tween 20 (TBST): Anti-p62 (cat. no. ab56416; Abcam; 1:1,000, anti-mouse), anti-LC3B (L7543; Sigma-Aldrich; Merck KGaA; 1:10,000; anti-rabbit), anti-ATG16L1 (cat. no. ab188642, Abcam; 1:1,000; anti-rabbit) and anti- $\beta$-actin (cat. no. ab8226; Abcam; 1:1,000; anti-mouse). After being washed with TBST three times, the membrane was incubated with horseradish peroxidase (HRP)-labeled goat anti-mouse immunoglobulin $\mathrm{G}$ ( $\mathrm{IgG} ; 1: 5,000$; cat. no. ab6789; Abcam) or goat anti-rabbit IgG (1:5,000; cat. no. ab6721; 
A
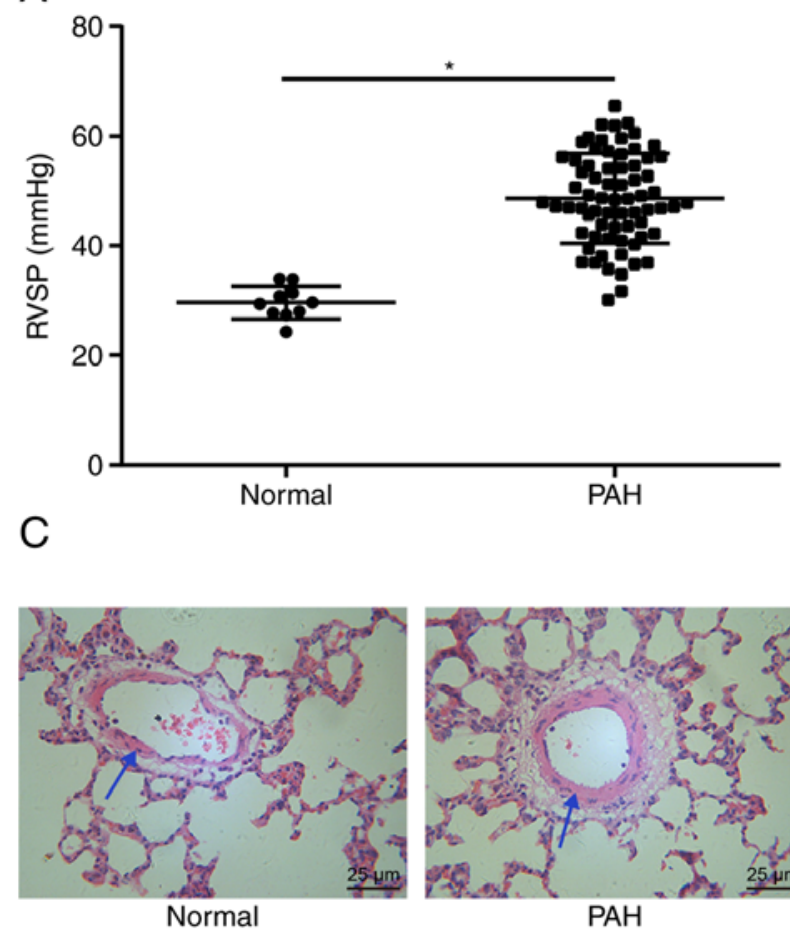

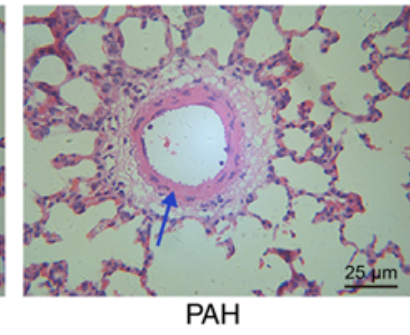

B
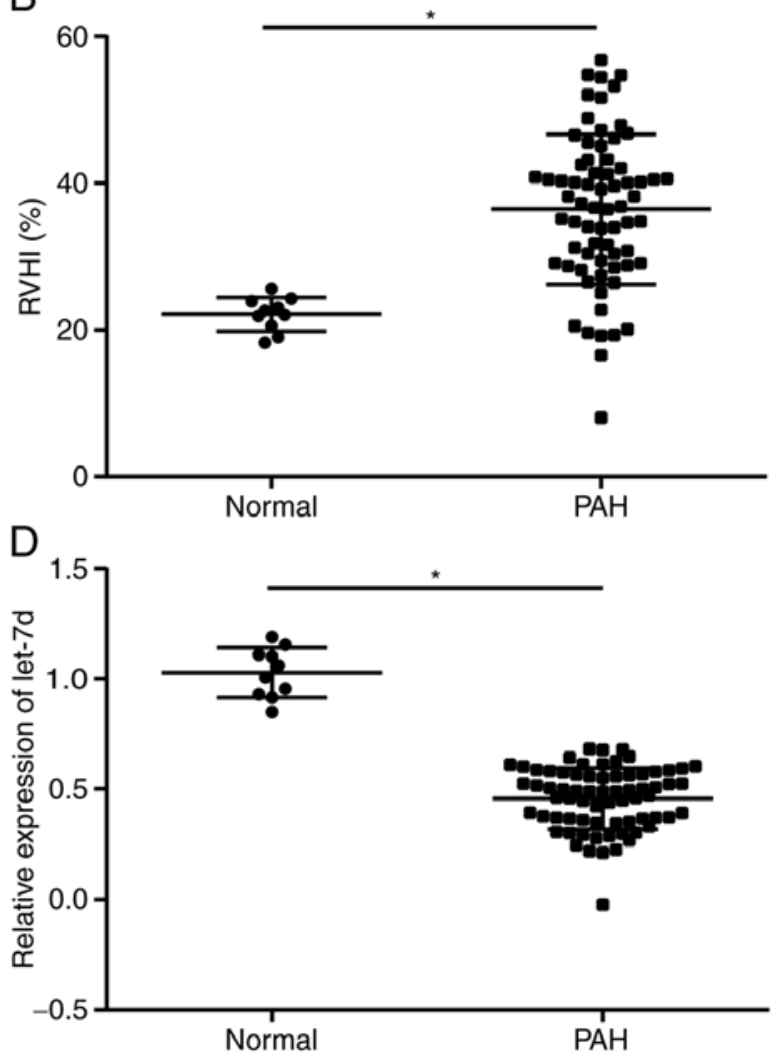

Figure 2. Rat models of PAH exhibit low expression of let-7d. (A) RVSP changes in rats in the normal and PAH groups. (B) RVHI changes in rats in the normal and PAH groups. (C) H\&E staining images of pulmonary blood vessels in rats in the normal and PAH groups; the blue arrow indicates the part with obvious changes. (D) The expression of let-7d in rats in the normal and PAH groups, as measured by reverse transcription-quantitative PCR. "P<0.05 vs. the normal group. The above data were all measurement data and expressed as the mean \pm standard deviation. t-tests were used for comparisons between two groups. Normal group, $n=10$; PAH group, $n=70$. RVSP, right ventricular systolic pressure; RVHI, right ventricular hypertrophy index; H\&E, hematoxylin and eosin; PAH, pulmonary arterial hypertension.

Abcam) secondary antibodies at room temperature for $1 \mathrm{~h}$. Following this step, the membrane was washed six times with TBST and visualized using enhanced chemiluminescence reagent (Thermo Fisher Scientific, Inc.). The gel image analysis software ImageJ (version 1.46; National Institute of Health) was employed to analyze the gray value of each band and the ratio of the gray value of the target protein band to that of the internal control protein band was calculated.

Immunofluorescence staining. The slides of human PAECs were prepared and then fixed with $40 \mathrm{~g} / \mathrm{l}$ polyformaldehyde at room temperature for $15 \mathrm{~min}$, washed three times with PBS, and sealed with $10 \%$ goat serum. Next, the slides were incubated overnight at $4^{\circ} \mathrm{C}$ with anti-LC3B (L7543; 1:100; Sigma-Aldrich, Merck KGaA; anti-rabbit) and anti-PECAM-1 (cat. no. sc-18916; 1:100; Santa Cruz Biotechnology, Inc.; anti-rat). After being washed with PBS three times, the tissues were subjected to further incubation with fluorescein isothiocyanate-labeled secondary antibodies (cat. no. Ab6717; 1:500; Abcam; anti-rabbit) and Cy3-labeled IgG secondary antibodies (cat. no. Ab6939; 1:500; Abcam; anti-rat) and incubated with protection from light for $60 \mathrm{~min}$ at room temperature. Confocal imaging was performed using an LSM510 Meta inverted microscope with a confocal laser scanning head (Carl Zeiss AG). Digital images were collected for analysis (SPOT; Diagnostic Instruments, Inc.). ImageJ (version 1.46; National
Institute of Health) was employed to analyze the fluorescence intensity and calculate the fluorescence intensity value.

ELISA. For analysis of rat plasma, $2 \mathrm{ml}$ blood samples were collected using blood collection tubes containing anticoagulants and then centrifuged at $600 \mathrm{x}$ g for $20 \mathrm{~min}$ at $4^{\circ} \mathrm{C}$, after which the supernatant was collected for detection. Rat lung tissues were extracted, washed with PBS, homogenized (with protease inhibitor) and centrifuged at $6,700 \mathrm{x} \mathrm{g}$ for $5-10 \mathrm{~min}$ at $4^{\circ} \mathrm{C}$, followed by collection of the supernatant. A rat endothelin-1 (ET-1) ELISA kit (CE-EL-R0167c) was purchased from Wuhan Elabscience Biotechnology Co., Ltd. Standard or sample $(100 \mu \mathrm{l})$ was added to each well and the plate was then incubated at $37^{\circ} \mathrm{C}$ for $90 \mathrm{~min}$ before the liquid was removed and the plate was dried. Then, $100 \mu \mathrm{l}$ biotinylated detection $\mathrm{Ab}$ was added to each well and the plate was incubated at $37^{\circ} \mathrm{C}$ for $60 \mathrm{~min}$, dried and washed three times. After that, $100 \mu \mathrm{l} \mathrm{HRP}$ was added to each well and the plate was incubated at $37^{\circ} \mathrm{C}$ for $30 \mathrm{~min}$, dried and washed five times. Substrate reagent $(90 \mu \mathrm{l})$ was then added to each well and the plate was incubated for $15 \mathrm{~min}$ at $37^{\circ} \mathrm{C}$ before $50 \mu \mathrm{l}$ stop solution was added to each well. The optical density (OD) value was then measured at $450 \mathrm{~nm}$. A standard curve was drawn with the standard protein concentrations on the X-axis and the OD (A) values on the Y-axis. The concentrations of ET-1 were obtained from the standard curve according to the OD values of the sample wells. 


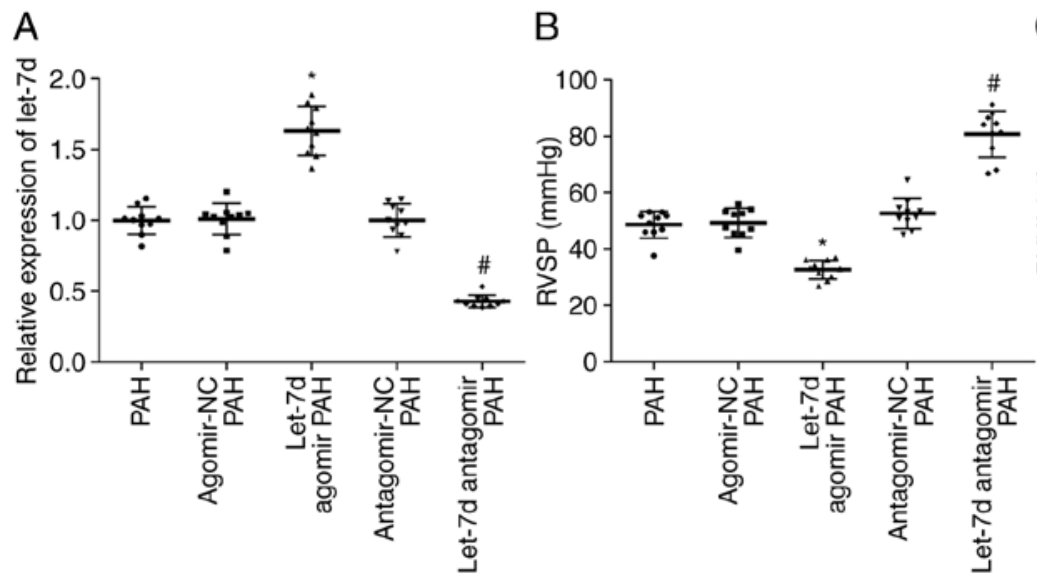

C
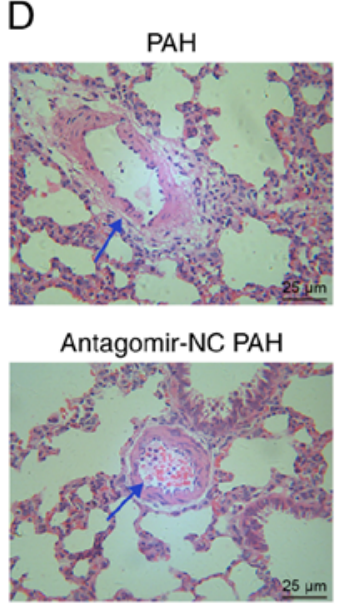

Agomir-NC PAH

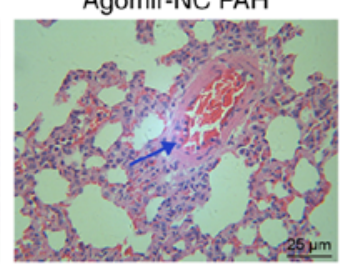

Let-7d antagomir PAH

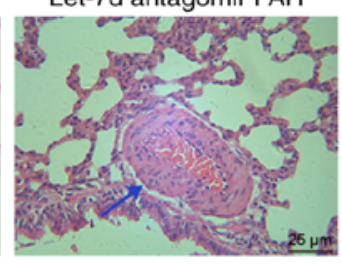

Let-7d agomir PAH
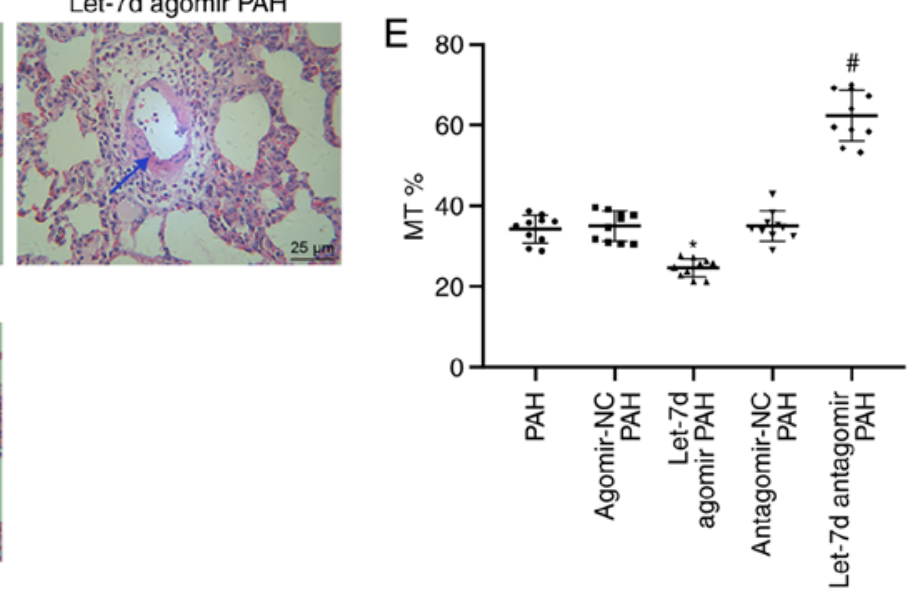

Figure 3. Let-7d ameliorates PAH. (A) The expression of let-7d in PAH rats injected with agomir or antagomir, as determined by reverse transcription-quantitative PCR. (B) RVSP changes in PAH rats injected with an agomir or antagomir. (C) RVHI changes in PAH rats injected with an agomir or antagomir. (D) H\&E staining images of rat lung tissues from PAH rats injected with the agomir or antagomir; the blue arrow indicates the part with obvious changes. (E) Percentage thickness of pulmonary vascular walls of rats. ${ }^{~} \mathrm{P}<0.05$ vs. the agomir NC PAH group. ${ }^{\# P}<0.05$ vs. the antagomir NC PAH group. The above data were all measurement data obeying normal distribution and expressed as the mean \pm standard deviation. One-way analysis of variance was used for comparisons among multiple groups, followed by a Tukey's post hoc test. Kruskal-Wallis $\mathrm{H}$ test was used for data with skewed distribution among multiple groups. N=10. RVSP, right ventricular systolic pressure; RVHI, right ventricular hypertrophy index; H\&E, hematoxylin and eosin; NC, negative control; PAH, pulmonary arterial hypertension; MT, myocardial thickness.

Statistical analysis. The experimental data were analyzed using SPSS 21.0 software (IBM Corp.). Enumeration data were analyzed using the Chi-square test. Measurement data were expressed as the mean \pm standard deviation. All data were subjected to normal distribution and homogeneity of variance tests. The measurement data conforming to normal distribution were expressed as the mean \pm standard deviation. The data with skewed distribution or heterogeneity of variance were expressed as the median \pm interquartile range. Data between two groups were compared using an unpaired t-test while data among multiple groups were analyzed using one-way analysis of variance, followed by a Tukey's post hoc test. The nonparametric Wilcoxon rank sum test was employed for data between two groups with a skewed distribution while Kruskal-Wallis $\mathrm{H}$ test was used for data among multiple groups with a skewed distribution. $\mathrm{P}<0.05$ was considered to indicate a statistically significant difference.

\section{Results}

Let-7d is poorly expressed in the plasma of PAH patients. The expression of let-7d in the plasma of the PAH group and the normal group was measured by RT-qPCR, and the results showed that compared with the plasma in the normal group, the plasma in the PAH group exhibited significantly reduced expression of let-7d $(\mathrm{P}<0.05$; Fig. 1). With the median let-7d expression as the dividing point, the $\mathrm{PAH}$ patients were classified as PAH patients with highly expressed let-7d or PAH patients with poorly expressed let-7d to analyze the relationship between let-7d expression and the clinicopathological features of PAH patients. The results (Table I) revealed that the expression of let-7d was not correlated with the age or gender of the PAH patients $(\mathrm{P}>0.05)$ but was significantly correlated with the cardiac function grade, degree of pulmonary hypertension and 6-min walk test performance $(\mathrm{P}<0.05)$. Collectively, the results indicate that the plasma of $\mathrm{PAH}$ patients displays reduced expression of let-7d compared with healthy subjects.

Let-7d is poorly expressed in the rat model of PAH. RVSP was measured by right cardiac catheterization. The RVHI was calculated to analyze right heart hypertrophy. Morphological changes in pulmonary vessels were observed by H\&E staining. RT-qPCR was performed to measure the expression of let-7d. 

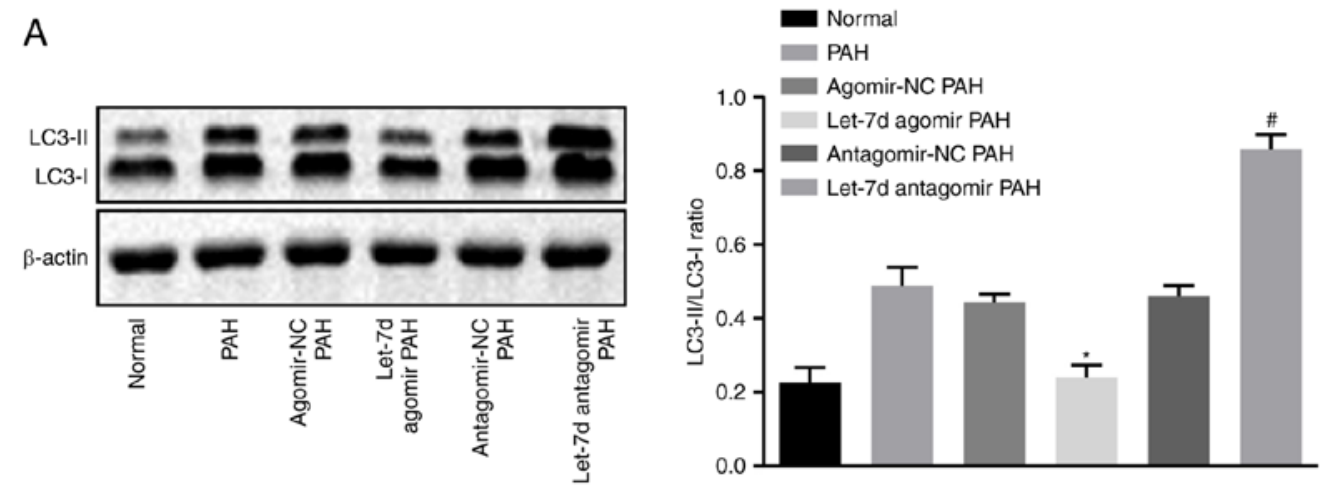

B
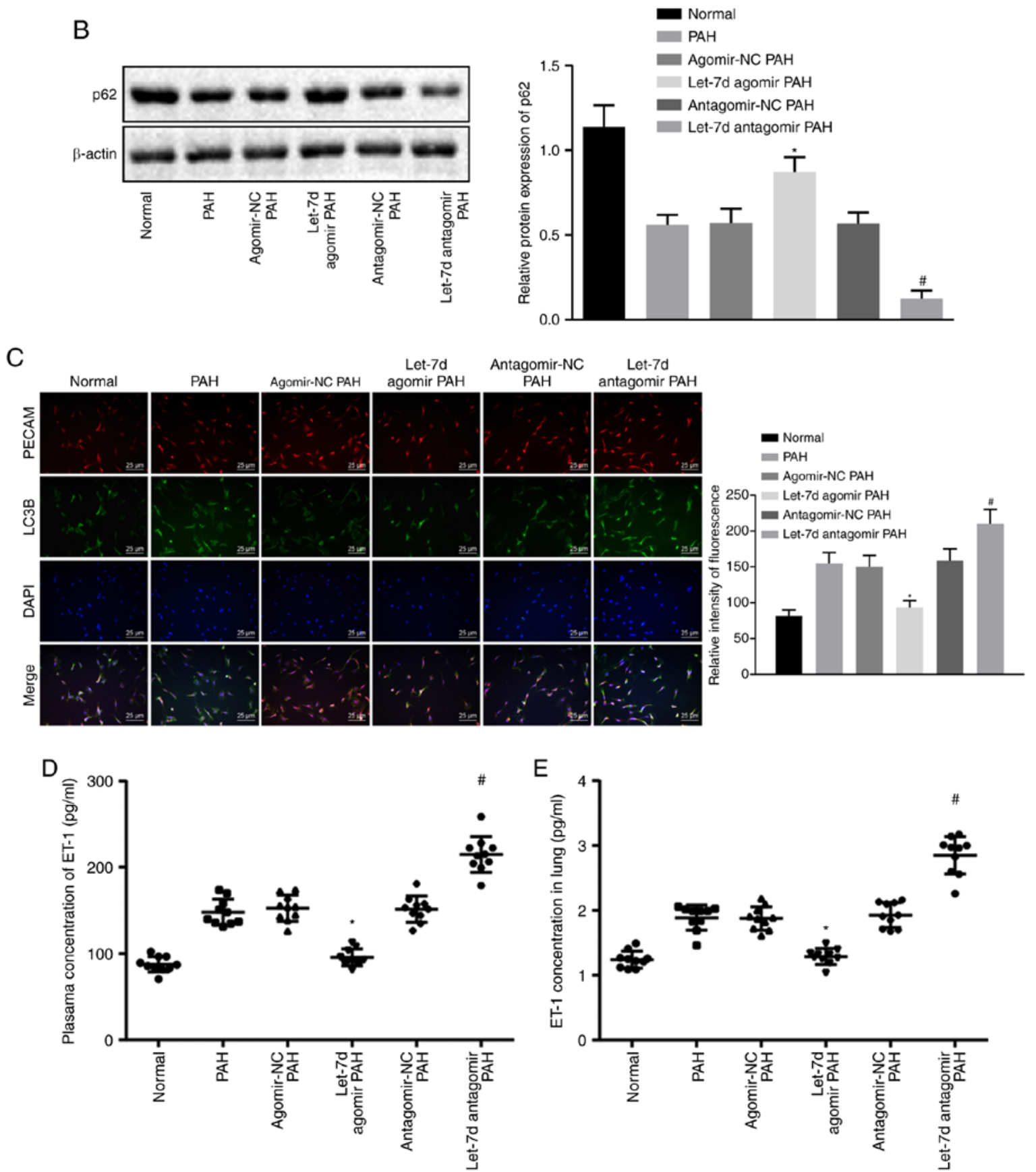

Figure 4. Let-7d inhibits autophagy and endothelin synthesis in PAECs. (A) The protein bands and levels of the autophagy-related protein LC3, as determined by western blot analysis. (B) The protein bands and levels of p62 in rat lung tissues, as measured by western blot analysis. Unprocessed blots are shown in Figure S1. (C) Quantification of the expression and relative fluorescence intensity of LC3B in PAECs, as assessed by immunofluorescence staining. (D) Levels of ET-1 in plasma, as determined by ELISA. (E) The levels of ET-1 in lung tissue, as measured by ELISA. "P<0.05 vs. the agomir NC PAH group. " $\mathrm{P}<0.05$ vs. the antagomir NC PAH group. The above data were all measurement data and expressed as the mean \pm standard deviation. One-way analysis of variance was used for comparisons among multiple groups, followed by a Tukey's post hoc test. N=10. PAECs, pulmonary artery endothelial cells; ET-1, endothelin-1; LC3, light chain 3; LC3B, light chain 3B; PAH, pulmonary arterial hypertension. 

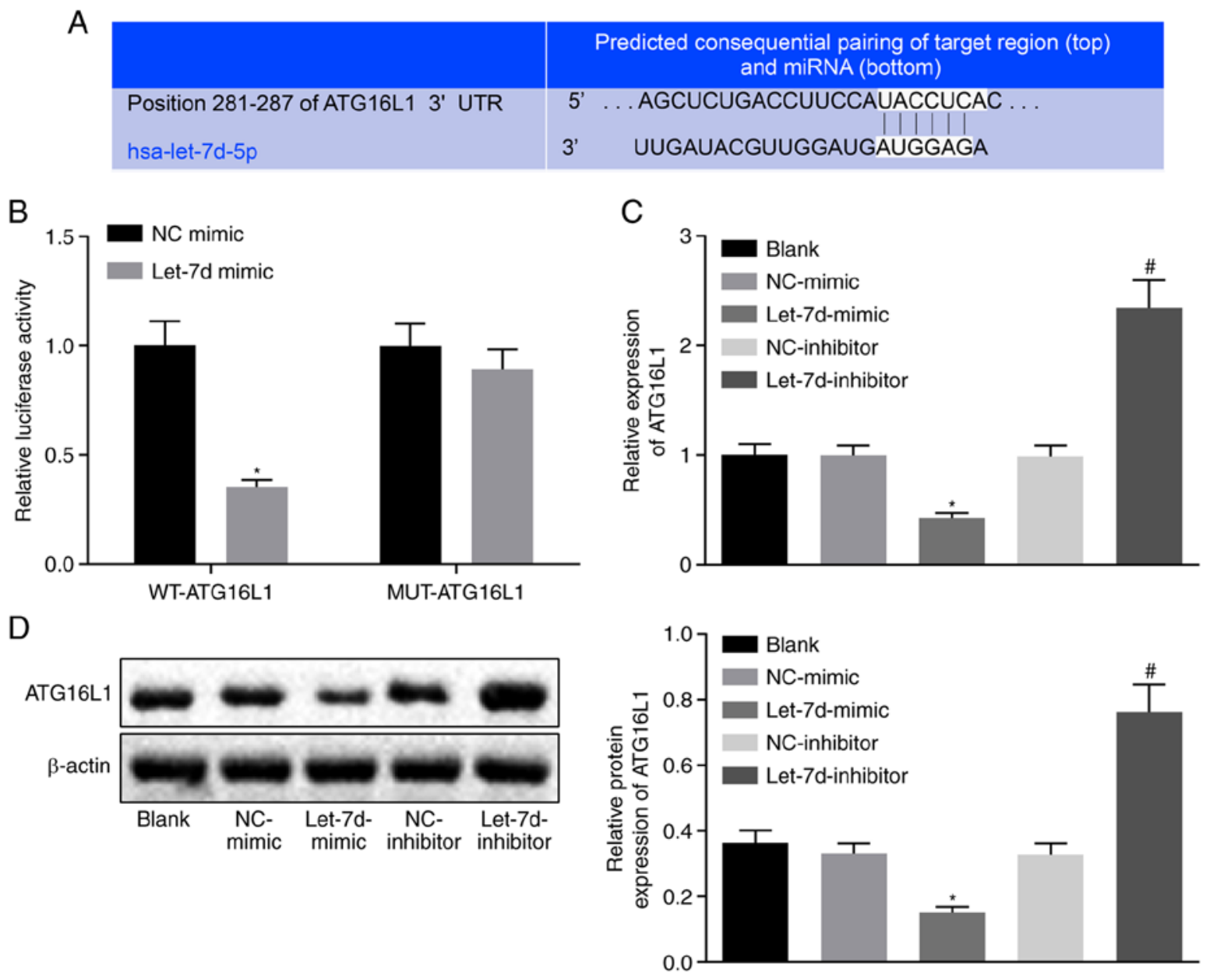

Figure 5. ATG16L1 is a target gene of let-7d. (A) The binding sites between let-7d and ATG16L1, as predicted with a bioinformatics website. (B) Luciferase activity, as detected by dual luciferase reporter gene assay. "P<0.05 vs. the WT-NC group. (C) The effect of let-7d on the expression of ATG16L1 in human PAECs. "P<0.05 vs. the NC mimic group. ${ }^{~} \mathrm{P}<0.05$ vs. the NC inhibitor group. (D) Quantification of the protein levels of ATG16L1 in human PAECs, as determined by western blot analysis. Unprocessed blots are shown in Figure $\mathrm{S} 1 .{ }^{*} \mathrm{P}<0.05$ vs. the $\mathrm{NC}$ mimic group. ${ }^{*} \mathrm{P}<0.05$ vs. the NC inhibitor group. The above data were all measurement data and expressed as the mean \pm standard deviation. One-way analysis of variance was used for comparisons among multiple groups, a Tukey's. The cell experiments were repeated three times. NC, negative control; ATG16L1, autophagy-related 16-like 1; WT, wild-type; MUT, mutant; UTR, untranslated region.

The results demonstrated that the PAH group showed significantly elevated RVSP and RVHI values ( $\mathrm{P}<0.05$; Fig. $2 \mathrm{~A}$ and $\mathrm{B})$, thicker pulmonary vascular walls $(34.65 \pm 3.58 \%)$ (Fig. $2 \mathrm{C})$ and significantly reduced let-7d expression (Fig. 2D) compared with the normal group $(22.12 \pm 2.31 \%$; $\mathrm{P}<0.05)$. The results above led to the conclusion that let-7d expression was low in rat models of $\mathrm{PAH}$.

Let-7d alleviates $P A H$. To further investigate the role of let-7d in PAH, RT-qPCR was employed to detect the expression of let-7d, right heart catheterization was performed to detect RVSP, RVHI values to assess cardiac hypertrophy and H\&E staining was performed to observe morphological changes in the pulmonary vessels. The results of RT-qPCR suggested that let-7d expression was significantly increased in PAH rats injected with let-7d agomir compared with PAH rats injected with agomir $\mathrm{NC}(\mathrm{P}<0.05)$, while the expression of let-7d was decreased in PAH rats injected with the let-7d antagomir compared with rats injected with the antagomir $\mathrm{NC}(\mathrm{P}<0.05$; Fig. 3A). Injection of let-7d agomir resulted in reduced RVSP and RVHI, while injection of the let-7d antagomir led to significantly elevated RVSP and RVHI values $(\mathrm{P}<0.05$; Fig. 3B and $\mathrm{C})$. The results of H\&E staining proved that PAH rats injected with let-7d agomir showed significantly thinner pulmonary vessel walls $(24.63 \pm 2.29 \%)$ than PAH rats injected with agomir NC $(34.97 \pm 3.72 \%$; $\mathrm{P}<0.05)$, while PAH rats injected with let-7d antagomir presented with significantly thicker pulmonary vessel walls $(62.40 \pm 6.33 \%)$ than $\mathrm{PAH}$ rats injected with antagomir $\mathrm{NC}(34.97 \pm 3.72 \% ; \mathrm{P}<0.05)$ (Fig. 3D and E). The findings above provided evidence that let-7d could relieve PAH.

Let-7d represses PAEC autophagy and endothelin synthesis. Previous studies have shown the presence of excessive autophagy (21) and ET-1 accumulation in PAH models (24). To further investigate the mechanism by which let-7d affects $\mathrm{PAH}$, western blot analysis was employed to assess the expression of autophagy-related proteins and immunofluorescence staining was employed to analyze the expression of LC3B, an autophagic marker, in PAECs. During autophagy, a small segment of the cytosolic polypeptide LC3 (LC3-I) was enzymatically cleaved, transforming the peptide into an 
A

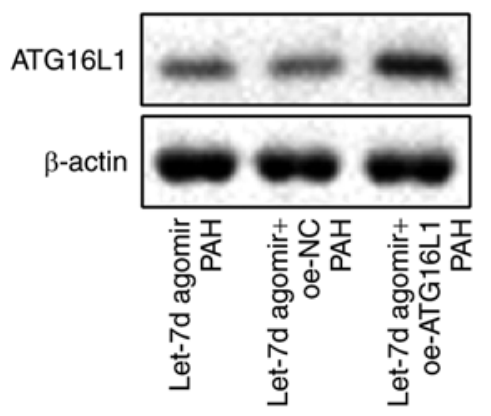

B

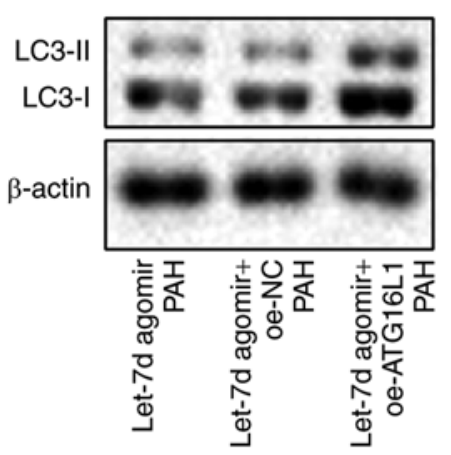

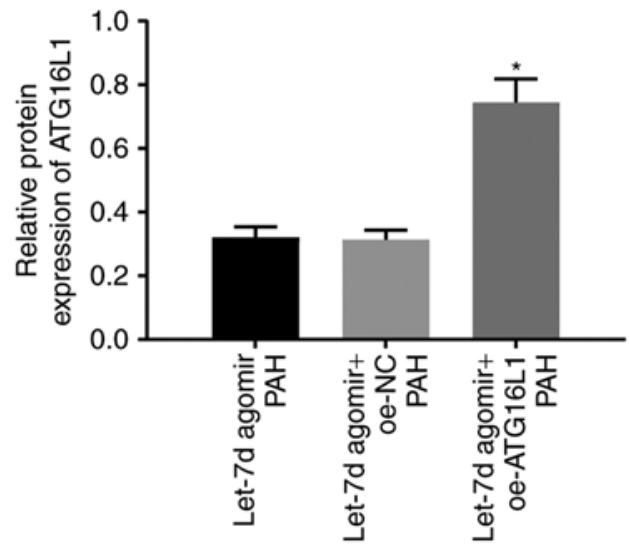

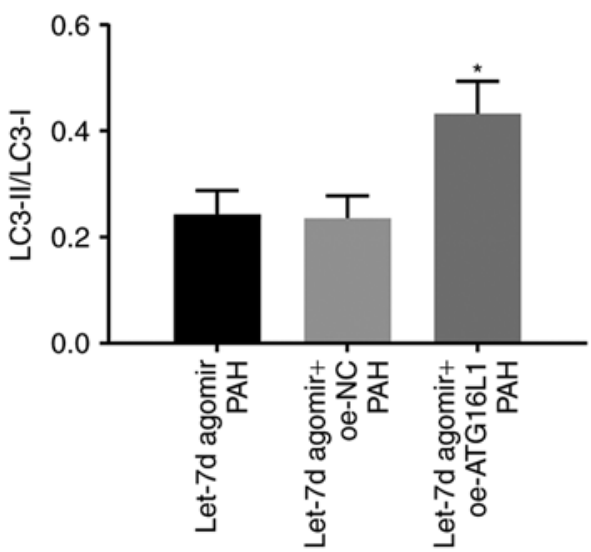

C

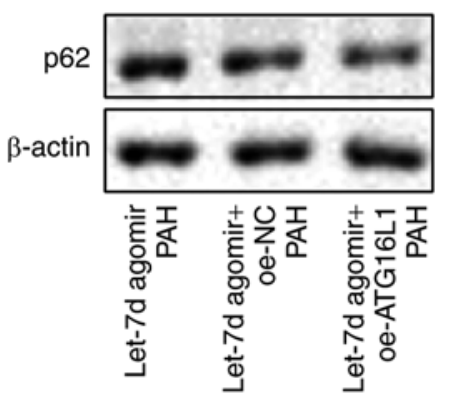

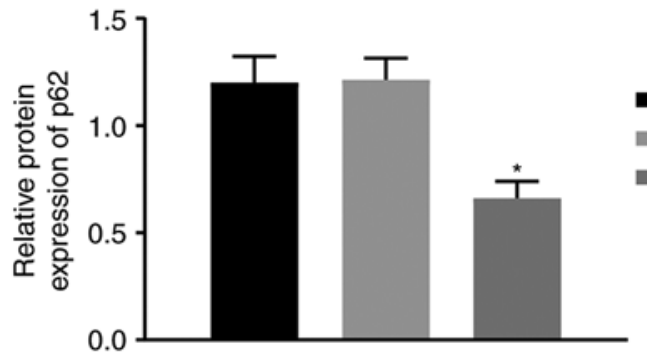

- Let-7d agomir PAH

- Let-7d agomir+oe-NC PAH

- Let-7d agomir+oe-ATG16L1 PAH

Figure 6. Let-7d suppresses autophagy in PAECs by targeting ATG16L1. (A) The protein bands and levels of ATG16L1, as determined by western blot analysis. (B) The protein bands and levels of LC3 in rat lung tissues, as measured by western blot analysis. (C) The protein bands and levels of p62 in rat lung tissues, as assessed by western blot analysis. Unprocessed blots are shown in Figure S1.

(autophagosome) membrane type (LC3-II). The ratio of LC3-II to LC3-I can be used to estimate the level of autophagy and LC3B can be used as a marker for autophagosomes. Western blot analysis showed that the expression of autophagy-related protein p62 was reduced and that the ratio of LC3-II to LC3-I was significantly increased in the PAH group compared with the normal group $(\mathrm{P}<0.05)$. Injection of let-7d agomir significantly elevated the levels of p62 and reduced the ratio of LC3-II to LC3-I compared with injection of agomir NC $(\mathrm{P}<0.05)$, while injection of the let-7d antagomir significantly decreased the levels of p62 and increased the ratio of LC3-II to LC3-I $(\mathrm{P}<0.05)$ compared with injection of the antagomir $\mathrm{NC}$ (Fig. 4A and B). Immunofluorescence staining showed that the expression of LC3B was significantly increased in the PAH group compared with in the normal group $(\mathrm{P}<0.05)$. The levels of LC3B were significantly decreased in rats injected with the let-7d agomir compared with in rats injected with the agomir
$\mathrm{NC}(\mathrm{P}<0.05)$. Injection of the let-7d antagomir significantly increased the levels of LC3B in comparison with injection of antagomir $\mathrm{NC}(\mathrm{P}<0.05$; Fig. 4C). The results of the ELISA, which was performed to detect the ET-1 concentrations in plasma and lung tissues, showed that the concentrations of ET-1 in plasma and lung tissues were significantly increased in the PAH group compared with in the normal group $(\mathrm{P}<0.05)$. Rats injected with the let-7d agomir presented with significantly decreased concentrations of ET-1 compared with those injected with agomir NC $(\mathrm{P}<0.05)$. Rats injected with the let-7d antagomir showed significantly elevated concentrations of ET-1 compared with those injected with the antagomir NC $(\mathrm{P}<0.05$; Fig. 4D and $\mathrm{E})$. In summary, let-7d inhibits autophagy and endothelin synthesis in PAECs.

Let-7d could directly target ATG16L1. The binding site of let-7d and ATG16L1 was predicted with a biological prediction 
D
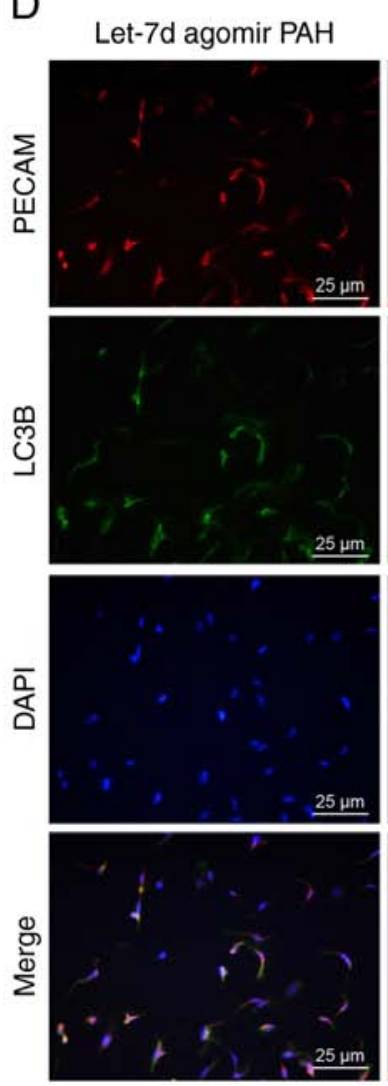
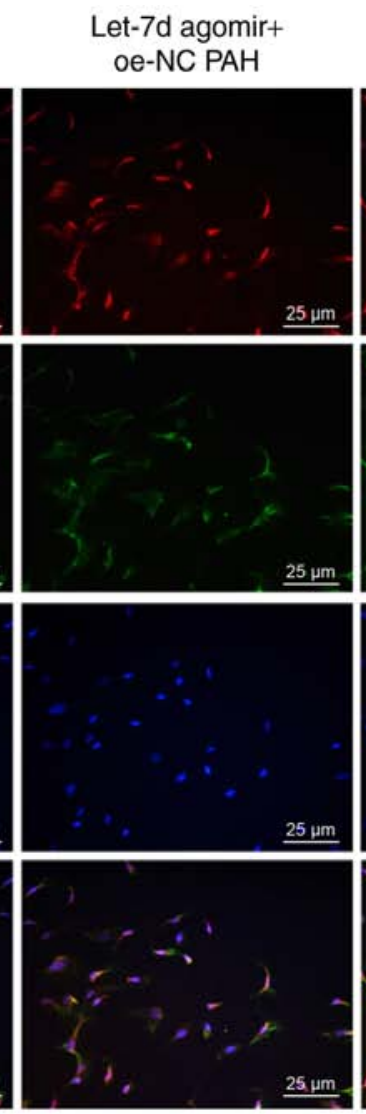

Let-7d agomir+ oe-ATG16L1 PAH
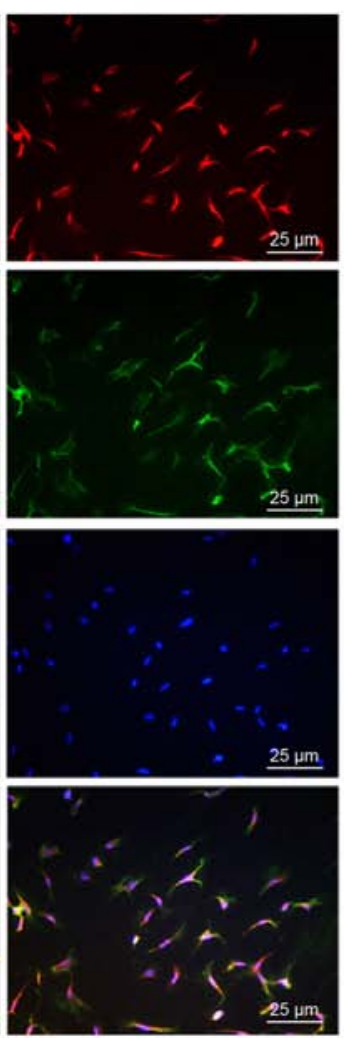

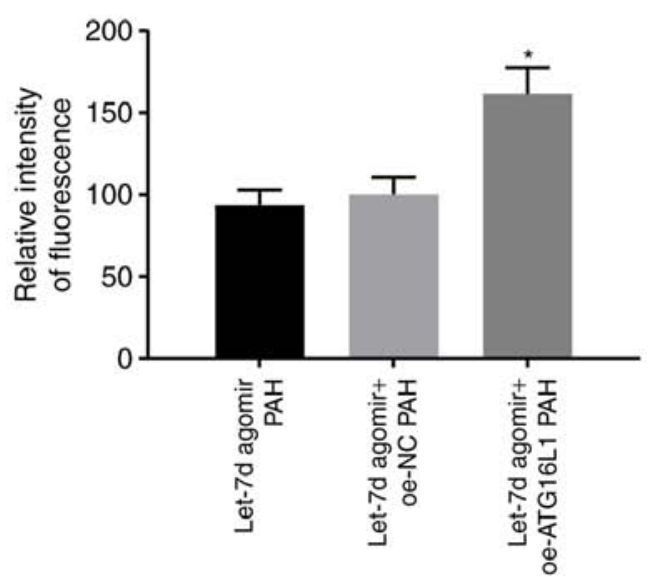

Figure 6. Continued. Let-7d suppresses autophagy in PAECs by targeting ATG16L1. (D) The relative fluorescence intensity and levels of LC3B in rat PAECs, as examined by immunofluorescence staining. ${ }^{*} \mathrm{P}<0.05$ vs. the let-7d agomir $\mathrm{PAH}+$ oe-NC group. The above data were all measurement data and expressed as the mean \pm standard deviation. One-way analysis of variance was used for comparisons among multiple groups. $\mathrm{n}=10$. NC, negative control; PAECs, pulmonary artery endothelial cells; PAH, pulmonary arterial hypertension; ATG16L1, autophagy-related 16-like 1; LC3, light chain 3; LC3B, light chain 3B; oe, overexpression.

website (Fig. 5A) and verified by luciferase reporter assay (Fig. 5B). The results suggested that the luciferase signal of the Wt-let-7d/ATG16L1 cotransfection group was significantly decreased in cells treated with the let-7d mimic compared with in cells treated with the $\mathrm{NC}$ mimic $(\mathrm{P}<0.05)$, while there was no significant difference in the luciferase activity of the Mut-let-7d/ATG16L1 3'UTR ( $P>0.05)$. In comparison with the cells treated with the $\mathrm{NC}$ plasmid, the cells treated with the let-7d mimic showed significantly reduced mRNA and protein expression of ATG16L1 $(\mathrm{P}<0.05)$, while the cells treated with the let-7d inhibitor presented significantly increased mRNA and protein expression of ATG16L1 ( $<<0.05$; Fig. 5C and D), suggesting that let-7d inhibited the expression of ATG16L1. The above results suggested that ATG16L1 is a target gene of let-7d and that let-7d could specifically bind to ATG16L1.

Let-7d inhibits autophagy in PAECs by targeting ATG16L1. To further investigate the effect of let-7d on autophagy in PAECs by targeting ATG16L1, western blot analysis was performed to measure the expression of autophagy-related proteins and immunofluorescence staining was employed to analyze the expression of LC3B in PAECs. As determined by western blot analysis, PAECs treated with the let-7d agomir and ATG16L1 overexpression vectors displayed significantly elevated levels of ATG16L1 and significantly elevated ratios of LC3-II to LC3-I but significantly reduced expression of p62 compared with PAECs treated with the let-7d agomir and oe-NC vectors $(\mathrm{P}<0.05$; Fig. 6A-C). The results of immunofluorescence staining showed injection of both let-7d agomir and the ATG16L1 overexpression vector significantly increased the levels of LC3B compared with injection of both let-7d agomir and the oe-NC vector $(\mathrm{P}<0.05$; Fig. $6 \mathrm{D})$. Based on these findings, let-7d could suppress autophagy in PAECs by targeting ATG16L1.

Let-7d alleviates PAH by inhibiting PAEC autophagy and endothelin synthesis through targeting of ATG16L1. To investigate the mechanism by which let-7d affects PAH by targeting ATG16L1, an ELISA was employed to detect the levels of ET-1 in plasma and lung tissues. Right heart catheterization was conducted to detect RVSP. The RVHI was analyzed to assess right heart hypertrophy. H\&E staining was performed to observe morphological changes in pulmonary vessels. The results of ELISA suggested that injection of both the let-7d agomir and the oe-ATG16L1 vector significantly elevated the ET-1 levels in the plasma and lungs versus injection of the let-7d agomir and oe-NC vector $(\mathrm{P}<0.05$; Fig. $7 \mathrm{~A}$ and $\mathrm{B})$. Rats treated with the let-7d agomir and oe-ATG16L1 vectors showed significantly increased RVSP and RVHI values compared with rats treated with both let-7d agomir and oe-NC vectors $(\mathrm{P}<0.05$; Fig. 7C and D). The results of $\mathrm{H} \& \mathrm{E}$ staining demonstrated that PAH rats injected with both let-7d agomir and oe-ATG16L1 vectors showed thicker medial walls of pulmonary blood 

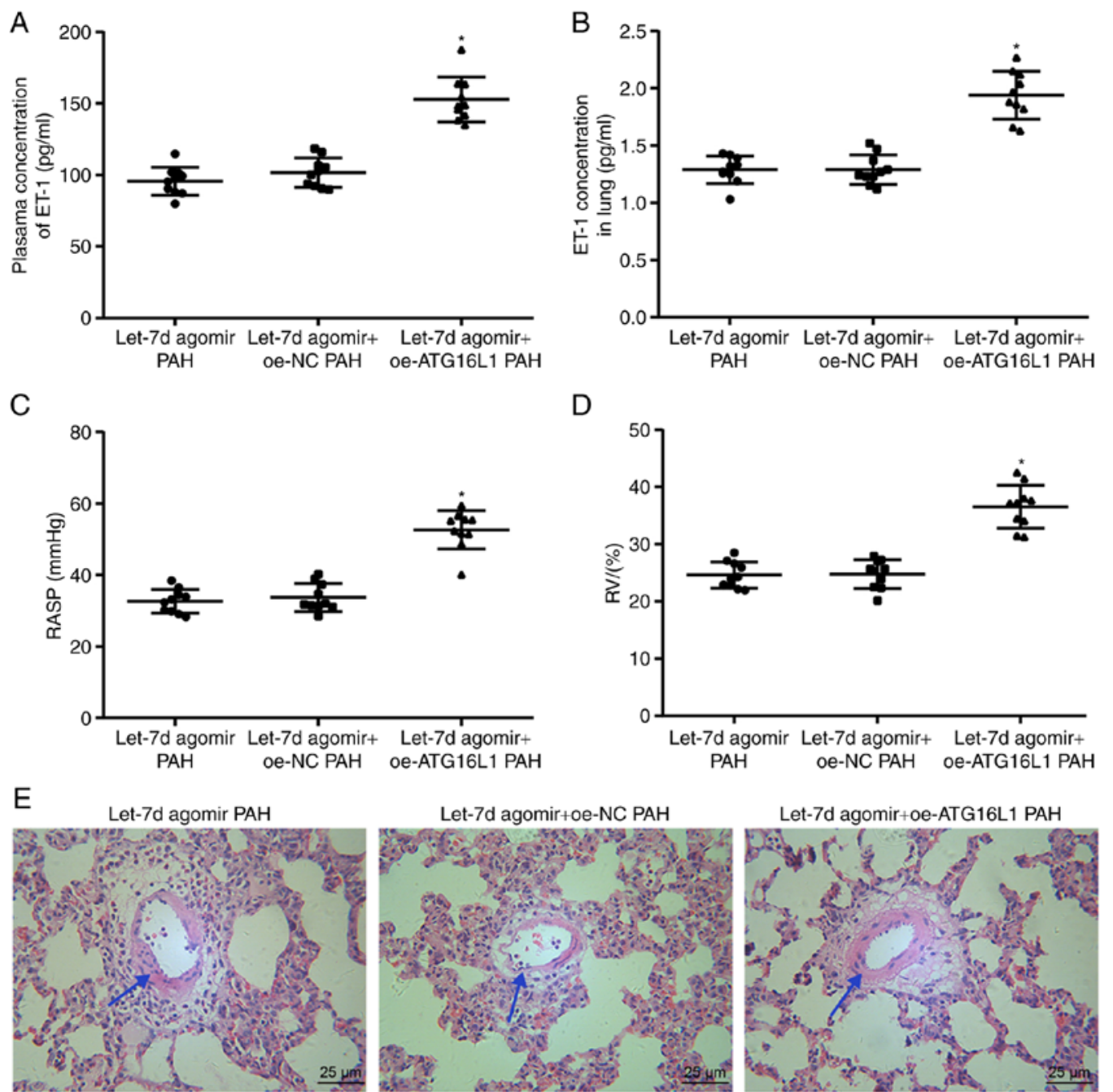

Let-7d agomir+oe-ATG16L1 PAH

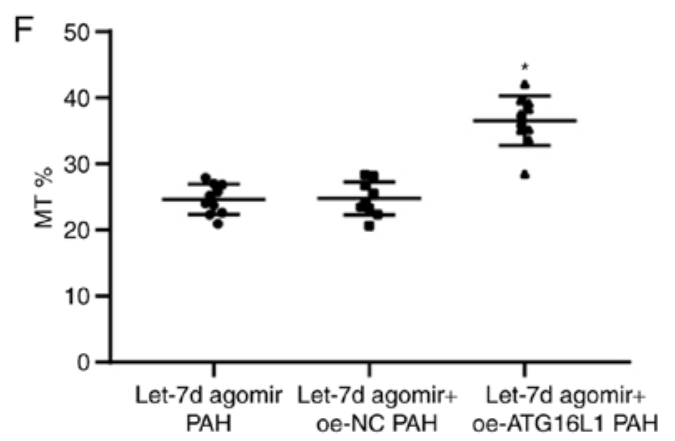

Figure 7. Let-7d ameliorates PAH via suppression of PAEC autophagy and endothelin synthesis through downregulation of ATG16L1. (A) The levels of ET-1 in rat plasma, as determined by ELISA. (B) The levels of ET-1 in rat lungs, as measured by ELISA. (C) RVSP changes in the rats in each group. (D) RVHI changes in the rats in each group. (E) Hematoxylin and eosin staining images of pulmonary arteries in the rats in each group; the arrow indicates the part with obvious changes. (F) Percentage thickness of pulmonary vascular wall of rats. ${ }^{*} \mathrm{P}<0.05$ vs. the let- $7 \mathrm{~d}$ agomir $\mathrm{PAH}+$ oe-NC group. The above data were all measurement data and expressed as mean \pm standard deviation. One-way analysis of variance was used for comparisons among multiple groups, followed by a Tukey's post hoc test. $\mathrm{n}=10$. NC, negative control; ET-1, endothelin-1; PAH, pulmonary arterial hypertension; ATG16L1, autophagy-related 16-like 1; RVSP, right ventricular systolic pressure; RVHI, right ventricular hypertrophy index; PAECs, pulmonary artery endothelial cells; oe, overexpression

vessels $(36.54 \pm 3.77 \%)$ than PAH rats injected with let-7d agomir and oe-NC vectors (24.78 $\pm 2.50 \%$; Fig. 7E). In conclusion, let-7d relieves PAH by reducing PAEC autophagy and endothelin synthesis through targeting of ATG16L1.

\section{Discussion}

PAH is a serious disease with features including vascular proliferation and remodeling of the pulmonary arteries that leads to a gradual elevation in pulmonary vascular resistance, right ventricular failure and ultimately death (25). Despite advances in treatment, PAH remains incurable with high morbidity and mortality rates (26). There is evidence showing that miRNAs function critically in the regulation of vascular remodeling in PAH (27). Thus, with the expectation to provide better treatment modalities for PAH patients, this study investigated the effects of let-7d on autophagy in PAECs and on endothelin synthesis. The results revealed that upregulation of 


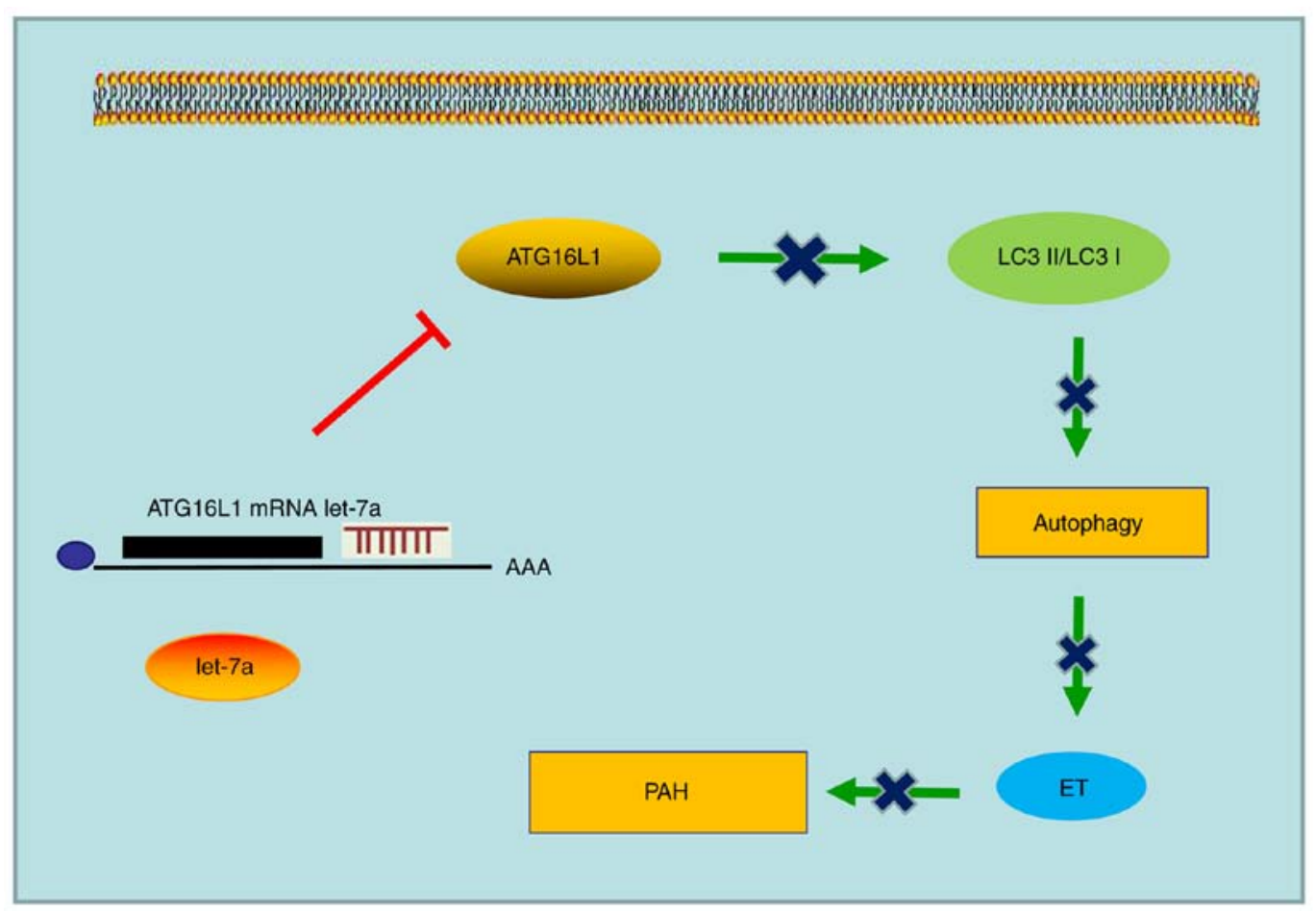

Figure 8. Molecular mechanism by which let-7d affects PAEC autophagy and ET synthesis in PAH. Let-7d inhibits PAEC autophagy and ET synthesis by binding to ATG16L1, thus alleviating PAH. PAH, pulmonary arterial hypertension; ATG16L1, autophagy-related 16-like 1; PAECs, pulmonary artery endothelial cells; ET, endothelin.

let-7d could downregulate ATG16L1 to inhibit autophagy in PAECs and suppress endothelin synthesis, thus ameliorating PAH.

The present study found that let-7d was poorly expressed in PAH and that upregulation of let-7d could inhibit PAEC autophagy and endothelin synthesis in PAH. Let-7d has been demonstrated to regulate the mesenchymal phenotypic properties of lung fibroblasts (28). The low expression of let-7d in idiopathic pulmonary fibrosis and the profibrotic effects of its downregulation indicate a critical role for let-7d in attenuating lung fibrosis (29). Notably, the let-7 family has been proven to be abnormally expressed in cardiovascular diseases, including cardiac fibrosis and hypertension; this family is expressed in human PAECs and is involved in the regulation of cardiovascular functions (13). For example, let-7d was found to be downregulated in cardiac fibroblasts in mice and its upregulation could mitigate fibrogenesis in cardiac fibrosis through regulation of platelet-activating factor receptors; cardiac fibrosis is an important feature of cardiovascular diseases (30). Wang et al (31) also demonstrated that let-7i was expressed at low levels in angiotensin II-infused hearts and that upregulation of let-7i could alleviate cardiac inflammation and fibrosis. Furthermore, downregulation of let- $7 \mathrm{c}$ has been reported in lung cancer and overexpression of let-7c has been shown to exert inhibitory effects on cell invasion, proliferation and migration (32). More importantly, let-7d has been found to be poorly expressed in patients with chronic thromboembolic pulmonary hypertension and this low expression can suppress the proliferation of PASMCs through upregulation of p21 (33).

Additionally, the present study demonstrated that let-7d could suppress PAEC autophagy and endothelin synthesis by negatively regulating ATG16L1. According to the current prediction analysis and luciferase activity determination, ATG16L1 is a target gene of let-7d, and let-7d can negatively regulate ATG16L1. It has previously been reported that various biological factors and chemical compounds are engaged in autophagy in vascular endothelial cells (VECs), and autophagy has potential effects on endothelial cells (34). There is evidence showing the involvement of miRNAs in autophagy through the regulation of ATGs or their regulators in human diseases (35). For example, miR-30d has been found to suppress autophagy in human cancer cells by inhibiting autophagy pathway-related genes, such as ATG12, ATG5 and ATG2; autophagosome formation; and the conversion of LC3B-I to LC3B-II (36). Furthermore, ATG16L1 plays an important role in autophagy and a previous study verified that suppression of autophagy by miR-410 overexpression in osteosarcoma cells was achieved partly through downregulation of ATG16L1 (17). Importantly, Xu et al (37) found that ATG4B is an underlying target gene of let-7i and that overexpression of let-7i could inhibit autophagic activity in preeclampsia through downregulation of ATG4B. Furthermore, endothelin is primarily released from VECs and forced expression of ET-1 has been found to be associated with elevations in right atrial pressure, pulmonary vascular resistance and mortality in patients with PAH (38). In addition, the expression of the ET-1 gene, which regulates the biological activities of ET-1, can be mediated by miRNA regulation (39). For instance, miR-125a, miR-125b and the let-7 family have been found to be upregulated in VECs, and both miR-125a and miR-125b can inhibit ET-1 expression in VECs by directly targeting preproET-1, which is essential for endothelin synthesis (40).

Taken together, the present results demonstrated that overexpression of let-7d could relieve PAH by inhibiting PAEC 
autophagy and endothelin synthesis through downregulation of ATG16L1 (Fig. 8). Thus, let-7d overexpression can serve as a potential therapeutic target for PAH and this study provides new insight for the treatment of PAH. Nevertheless, more studies are needed to analyze the specificity and sensitivity of this molecular tool as a biomarker of PAH.

\section{Acknowledgements}

Not applicable.

\section{Funding}

The present study was supported by the General Program of National Natural Science Foundation (grant no. 81871187), the Regional Projects of National Natural Science Foundation (grant no.81460239) and the Natural Science FoundationProject of Ningxia Hui Autonomous Region (grant no. NZ17195).

\section{Availability of data and materials}

The datasets generated and/or analyzed during the current study are available from the corresponding author on reasonable request.

\section{Authors' contributions}

MO designed the study. XL collated the data. SC and SZ carried out data analyses and produced the initial draft of the manuscript. JT participated in the design, interpretation of the results and contributed to drafting the manuscript. All authors read and approved the final manuscript.

\section{Ethics approval and consent to participate}

The current study was approved by the Ethics Committee and the Experimental Animal Ethics Committee of Qingdao Municipal Hospital. Written informed consent was obtained from all participants prior to the study. The animal experiment strictly adhered to the principles of using the least number of animals to complete the experiment and minimizing the pain of the experimental animals.

\section{Patient consent for publication}

Not applicable.

\section{Competing interests}

The authors declare that they have no competing interests.

\section{References}

1. Steele P, Strange G, Wlodarczyk J, Dalton B, Stewart S, Gabbay E and Keogh A: Hemodynamics in pulmonary arterial hypertension $(\mathrm{PAH})$ : Do they explain long-term clinical outcomes with PAH-specific therapy? BMC Cardiovasc Disord 10: 9, 2010.

2. Preston IR, Roberts KE, Miller DP, Sen GP, Selej M, Benton WW, Hill NS and Farber HW: Effect of warfarin treatment on survival of patients with pulmonary arterial hypertension $(\mathrm{PAH})$ in the registry to evaluate early and long-term $\mathrm{PAH}$ disease management (REVEAL). Circulation 132: 2403-2411, 2015.
3. Sikirica M, Iorga SR, Bancroft T and Potash J: The economic burden of pulmonary arterial hypertension (PAH) in the US on payers and patients. BMC Health Serv Res 14: 676, 2014.

4. Mehta J, Parthasarathy PT, Lockey R and Kolliputi N: New hope for a microRNA therapy for pulmonary arterial hypertension. Front Genet 4: 137, 2013.

5. Rubin LJ, Galie N, Grimminger F, Grunig E, Humbert M, Jing ZC, Keogh A, Langleben D, Fritsch A, Menezes F, et al: Riociguat for the treatment of pulmonary arterial hypertension: A long-term extension study (PATENT-2). Eur Respir J 45: 1303-1313, 2015.

6. Meloche J, Le Guen M, Potus F, Vinck J, Ranchoux B, Johnson I, Antigny F, Tremblay E, Breuils-Bonnet S, Perros F, et al: MiR-223 reverses experimental pulmonary arterial hypertension. Am J Physiol Cell Physiol 309: C363-C372, 2015.

7. Potus F, Graydon C, Provencher S and Bonnet S: Vascular remodeling process in pulmonary arterial hypertension, with focus on miR-204 and miR-126 (2013 grover conference series). Pulm Circ 4: 175-184, 2014

8. Jiang L, Wang Y, Rong Y, Xu L, Chu Y, Zhang Y and Yao Y: MiR-1179 promotes cell invasion through SLIT2/ROBO1 axis in esophageal squamous cell carcinoma. Int J Clin Exp Pathol 8: 319-327, 2015.

9. Courboulin A, Paulin R, Giguere NJ, Saksouk N, Perreault T, Meloche J, Paquet ER, Biardel S, Provencher S, Cote J, et al: Role for miR-204 in human pulmonary arterial hypertension. J Exp Med 208: 535-548, 2011.

10. Stevens HC, Deng L, Grant JS, Pinel K, Thomas M, Morrell NW, MacLean MR, Baker AH and Denby L: Regulation and function of miR-214 in pulmonary arterial hypertension. Pulm Circ 6: 109-117, 2016.

11. Ramberg H, Alshbib A, Berge V, Svindland A and Tasken KA: Regulation of PBX3 expression by androgen and Let-7d in prostate cancer. Mol Cancer 10: 50, 2011.

12. Dai X and Cai Y: Down-regulation of microRNA let-7d inhibits the proliferation and invasion of trophoblast cells in preeclampsia. J Cell Biochem 119: 1141-1151, 2018.

13. Bao MH, Feng X, Zhang YW, Lou XY, Cheng Y and Zhou HH: Let-7 in cardiovascular diseases, heart development and cardiovascular differentiation from stem cells. Int J Mol Sci 14: 23086-23102, 2013.

14. Han J, Pan XY, Xu Y, Xiao Y, An Y, Tie L, Pan Y and Li XJ: Curcumin induces autophagy to protect vascular endothelial cell survival from oxidative stress damage. Autophagy 8: 812-825, 2012.

15. Kim JK, Yuk JM, Kim SY, Kim TS, Jin HS, Yang CS and Jo EK: MicroRNA-125a inhibits autophagy activation and antimicrobial responses during mycobacterial infection. J Immunol 194: 5355-5365, 2015

16. Nishimura T, Kaizuka T, Cadwell K, Sahani MH, Saitoh T, Akira S, Virgin HW and Mizushima N: FIP200 regulates targeting of Atg16L1 to the isolation membrane. EMBO Rep 14: 284-291, 2013.

17. Chen R, Li X, He B and Hu W: MicroRNA-410 regulates autophagy-related gene ATG16L1 expression and enhances chemosensitivity via autophagy inhibition in osteosarcoma. Mol Med Rep 15: 1326-1334, 2017.

18. Magne J, Gustafsson P, Jin H, Maegdefessel L, Hultenby K, Wernerson A, Eriksson P, Franco-Cereceda A, Kovanen PT, Goncalves I and Ehrenborg E: ATG16L1 expression in carotid atherosclerotic plaques is associated with plaque vulnerability. Arterioscler Thromb Vasc Biol 35: 1226-1235, 2015.

19. Chin KM, Rubin LJ, Channick R, Di Scala L, Gaine S, Galie N, Ghofrani HA, Hoeper MM, Lang IM, McLaughlin VV, et al: Association of $\mathrm{N}$-terminal pro brain natriuretic peptide and long-term outcome in patients with pulmonary arterial hypertension. Circulation 139: 2440-2450, 2019.

20. Ruiz-Irastorza G, Garmendia M, Villar I, Egurbide MV and Aguirre C: Pulmonary hypertension in systemic lupus erythematosus: Prevalence, predictors and diagnostic strategy. Autoimmun Rev 12: 410-415, 2013.

21. Zhai C, Shi W, Feng W, Zhu Y, Wang J, Li S, Yan X, Wang Q, Zhang Q, Chai L, et al: Activation of AMPK prevents monocrotaline-induced pulmonary arterial hypertension by suppression of NF-kappaB-mediated autophagy activation. Life Sci 208: 87-95, 2018.

22. Lee SJ, Smith A, Guo L, Alastalo TP, Li M, Sawada H, Liu X, Chen $\mathrm{ZH}$, Ifedigbo E, Jin Y, et al: Autophagic protein LC3B confers resistance against hypoxia-induced pulmonary hypertension. Am J Respir Crit Care Med 183: 649-658, 2011. 
23. Livak KJ and Schmittgen TD: Analysis of relative gene expression data using real-time quantitative PCR and the 2(-Delta Delta C(T)) method. Methods 25: 402-408, 2001.

24. Montani D, Souza R, Binkert C, Fischli W, Simonneau G, Clozel M and Humbert M: Endothelin-1/endothelin-3 ratio: A potential prognostic factor of pulmonary arterial hypertension. Chest 131: 101-108, 2007.

25. Humbert M, Sitbon O, Chaouat A, Bertocchi M, Habib G, Gressin V, Yaici A, Weitzenblum E, Cordier JF, Chabot F, et al: Survival in patients with idiopathic, familial, and anorexigen-associated pulmonary arterial hypertension in the modern management era. Circulation 122: 156-163, 2010.

26. Lee WT, Ling Y, Sheares KK, Pepke-Zaba J, Peacock AJ and Johnson MK: Predicting survival in pulmonary arterial hypertension in the UK. Eur Respir J 40: 604-611, 2012.

27. Caruso P, Dempsie Y, Stevens HC, McDonald RA, Long L, Lu R, White K, Mair KM, McClure JD, Southwood M, et al: A role for miR-145 in pulmonary arterial hypertension: Evidence from mouse models and patient samples. Circ Res 111: 290-300, 2012

28. Huleihel L, Ben-Yehudah A, Milosevic J, Yu G, Pandit K, Sakamoto K, Yousef H, LeJeune M, Coon TA, Redinger CJ, et al: Let-7d microRNA affects mesenchymal phenotypic properties of lung fibroblasts. Am J Physiol Lung Cell Mol Physiol 306: L534-L542, 2014.

29. Pandit KV, Corcoran D, Yousef H, Yarlagadda M, Tzouvelekis A, Gibson KF, Konishi K, Yousem SA, Singh M, Handley D, et al: Inhibition and role of let-7d in idiopathic pulmonary fibrosis. Am J Respir Crit Care Med 182: 220-229, 2010.

30. Liang H, Pan Z, Zhao X, Liu L, Sun J, Su X, Xu C, Zhou Y, Zhao D, Xu B, et al: LncRNA PFL contributes to cardiac fibrosis by acting as a competing endogenous RNA of let-7d. Theranostics 8: 1180-1194, 2018.

31. Wang X, Wang HX, Li YL, Zhang CC, Zhou CY, Wang L, Xia YL, Du J and Li HH: MicroRNA Let-7i negatively regulates cardiac inflammation and fibrosis. Hypertension 66: 776-785, 2015.
32. Zhao B, Han H, Chen J, Zhang Z, Li S, Fang F, Zheng Q, Ma Y, Zhang J, Wu N and Yang Y: MicroRNA let-7c inhibits migration and invasion of human non-small cell lung cancer by targeting ITGB3 and MAP4K3. Cancer Lett 342: 43-51, 2014.

33. Wang L, Guo LJ, Liu J, Wang W, Yuan JX, Zhao L, Wang J and Wang C: MicroRNA expression profile of pulmonary artery smooth muscle cells and the effect of let-7d in chronic thromboembolic pulmonary hypertension. Pulm Circ 3: 654-664, 2013.

34. Jiang F: Autophagy in vascular endothelial cells. Clin Exp Pharmacol Physiol 43: 1021-1028, 2016.

35. Guo L, Zhao J, Qu Y, Yin R, Gao Q, Ding S, Zhang Y, Wei J and Xu G: MicroRNA-20a inhibits autophagic process by targeting ATG7 and ATG16L1 and favors mycobacterial survival in macrophage cells. Front Cell Infect Microbiol 6: 134, 2016.

36. Yang X, Zhong X, Tanyi JL, Shen J, Xu C, Gao P, Zheng TM, DeMichele A and Zhang L: Mir-30d regulates multiple genes in the autophagy pathway and impairs autophagy process in human cancer cells. Biochem Biophys Res Commun 431: 617-622, 2013.

37. Xu Y, Huang X, Xie J, Chen Y, Fu J and Wang L: Let-7i-induced Atg4B suppression is essential for autophagy of placental trophoblast in preeclampsia. J Cell Physiol 232: 2581-2589, 2017.

38. Shao D, Park JE and Wort SJ: The role of endothelin-1 in the pathogenesis of pulmonary arterial hypertension. Pharmacol Res 63: 504-511, 2011.

39. Jacobs ME, Wingo CS and Cain BD: An emerging role for microRNA in the regulation of endothelin-1. Front Physiol 4: 22, 2013.

40. Li D, Yang P, Xiong Q, Song X, Yang X, Liu L, Yuan W and Rui YC: MicroRNA-125a/b-5p inhibits endothelin-1 expression in vascular endothelial cells. J Hypertens 28: 1646-1654, 2010.

This work is licensed under a Creative Commons Attribution-NonCommercial-NoDerivatives 4.0 International (CC BY-NC-ND 4.0) License. 